\title{
A step closer to combating acquired resistance in CRC
}

Patients with metastatic colorectal cancer (CRC) who show dramatic and rapid responses to targeted agents inevitably develop resistance. Not much is known about why these responses are short lived and why tumours rapidly regrow and become resistant to further treatment. Now, two articles published in Nature bring us closer to defining the molecular basis of acquired resistance and allow its non-invasive detection.

Alberto Bardelli and colleagues wanted to identify potential mechanisms of cetuximab resistance. "We initially generated cetuximab-resistant variants of two CRC cellular models that are highly sensitive to EGFR inhibition," explains Bardelli. They confirmed that pre-existing KRAS amplified or mutant clones were causally associated with the onset of acquired resistance to cetuximab. Next, they examined tumour biopsies from 10 patients with CRC who developed resistance to cetuximab or panitumumab treatment. "Next-generation sequencing identified KRAS mutations only in the biopsies obtained after the patients had received anti-EGFR therapies." Bardelli's team reasoned that detection of mutant KRAS in the blood of patients treated with cetuximab might allow early identification of resistance before radiographic evidence of disease progression. Remarkably, analysis of plasma samples from patients treated with cetuximab confirmed the same KRAS variants present in post-treatment biopsies as early as 10 months before disease progression. Bardelli explains the significance of their findings, "it is now possible to monitor the evolution of the tumour in response to therapy using a blood draw to detect early mutations that will cause resistance."

In the second study, Luis Diaz and researchers assessed circulating tumour DNA (ctDNA) fragments in the blood of patients with CRC, using two digital PCRbased approaches, to analyse the tumour genotype during panitumumab therapy. Diaz explains the methodology, "we adopted this approach as ctDNA can provide a snapshot into the comprehensive genetic status of patients with metastatic disease; the study we conducted in essence assessed all the indivual mutations at once." Diaz and his team confirmed that resistance mutations are present before treatment, and that the "number of resistant cells present before EGFR blockade are very low and are not detectable by current sequencing technology".

Both studies reveal that resistance to EGFR-targeted agents is a fait accompli and an inevitable

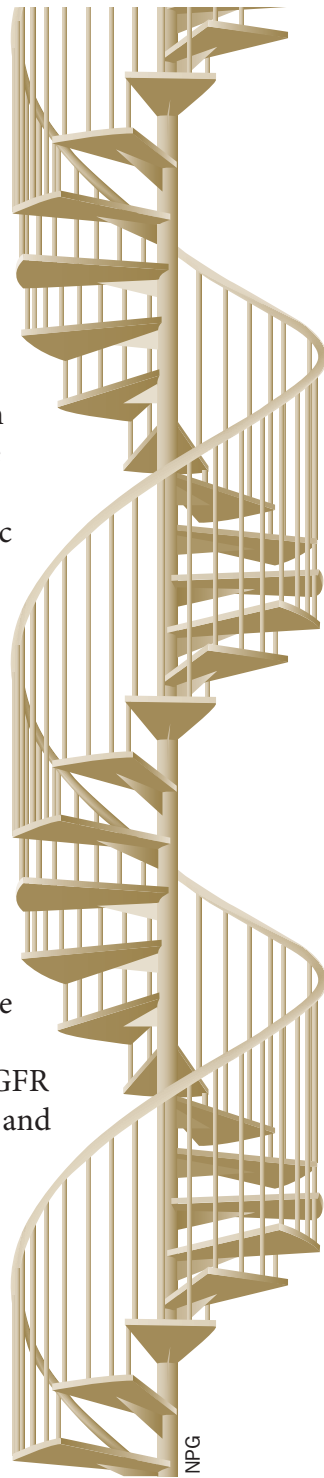
consequence of highly targeted singleagent therapy. Bardelli notes the most exciting finding of his work is, "the possibility of exploiting the liquid biopsy approach may allow future clinical trials to be initiated as soon as the resistant clones emerge."

\section{Lisa Hutchinson}

Original articles Misale, S. et al. Emergence of KRAS mutations and acquired resistance to anti-EGFR therapy in colorectal cancer. Nature doi:10.1038/ nature11156|Diaz, L. A. et al. The molecular evolution of acquired resistance to targeted EGFR blockade in colorectal cancers. Nature doi:10.1038/nature 11219 